Original Article

\title{
FETAL LIVER MORPHOMETRY AT DIFFERENT CROWN RUMP LENGTHS
}

\author{
Anamika Jaiswal ${ }^{\star}$, Ankit Kaushik ${ }^{\star \star}$, Jayanti Pant ${ }^{\star \star \star}$, AK Singh $^{\star}$, MK Pant ${ }^{\star \star \star \star}$ \\ *Department of Anatomy, GMC, Haldwani, Uttarakhand, India \\ ${ }^{* *}$ Department of Pathology, GMC, Haldwani, Uttarakhand, India \\ ${ }^{* * *}$ Department of Physiology, AlIMS, Rishikesh, Uttarakhand, India \\ ****Department of Anatomy, Government Doon Medical College, Dehradun, Uttarakhand, India
}

\begin{abstract}
Introduction: Normal morphometric parameters of liver in human fetus were studied at various stage of development with an aim to get insight into the normal development of fetal liver.
\end{abstract}

Material \& Methods: Dissection of 29 human fetuses was performed. Anthropometric study was done and various parameters were measured.

Results: The development of liver is associated with increase in all the parameters although the increase is highly variable in different gestational age, indicating that liver development showed characteristic but highly regulated development to reach its fully developed stage.

Conclusion: The present study will be helpful in understanding the normal fetal liver development.

Keywords: Fetus, liver, development, anthropometry.

\section{INTRODUCTION}

Fetal liver is one of the most dynamic organs during gestational life arising from endodermal evagination of foregut and septum transversum mesenchyme [1]. It performs essential functions like haematopoiesis in fetus [2]. Fetal liver size is an important parameter of growth, showing linear increase in the lobes of liver during gestational period [3]. Liver growth gets deranged in numerous conditions. Large liver for gestational age was found in numerous conditions like gestational diabetes, isoimmunization and fetal anemias, intrauterine infections, fetal heart failure, tumours, certain metabolic diseases and fetal macrosomias [3,4]. Intrauterine growth retardation (IUGR) is associated with markedly retarded liver growth in comparison to other organs [2]. Since liver growth is a good indicator of fetal growth and outcome of pregnancy, our aim is to find out the development of fetal liver grossly, studying its various parameter [5] and contribute to available literature in understanding the liver development during gestation period.

\section{MATERIAL AND METHODS}

This prospective study was conducted in the Department of Anatomy, Government Medical College, Haldwani. Twenty nine dead fetuses with no external anomaly were obtained from Obstetrics and Gynecology Department, Dr Sushila Tiwari hospital over a period of two years (period commencing from 2011 to 2013), with due regard on ethical ground. Fetuses were preserved in formalin and various gross features were studied as per study design. The crown rump length (CRL) of the fetus was recorded. Fetuses were dissected and liver was displayed (Fig. 1). Various measurements were recorded using measuring tape, compass and vernier caliper in centimeter $(\mathrm{cm})$ rounded off to second decimal place. The liver was exposed by making transverse incision extending from the umbilicus to the mid axillary line bilaterally, and two vertical incisions, extending from the costal arch to the iliac crest alongside the mid axillary line bilaterally [5]. 
Liver was observed for any gross and congenital anomalies. The maximum height of right and left hepatic lobes were taken. The maximum transverse diameter of liver was taken along with maximum transverse length of right and left lobe of liver (Fig. 2). The liver was removed by dissecting inferior vena cava and excising ligaments of the liver and after that its weight was recorded before embalming. The length and breadth of quadrate and caudate lobe were also measured. The length and breadth of porta hepatis were measured too. The results were tabulated and analysed.

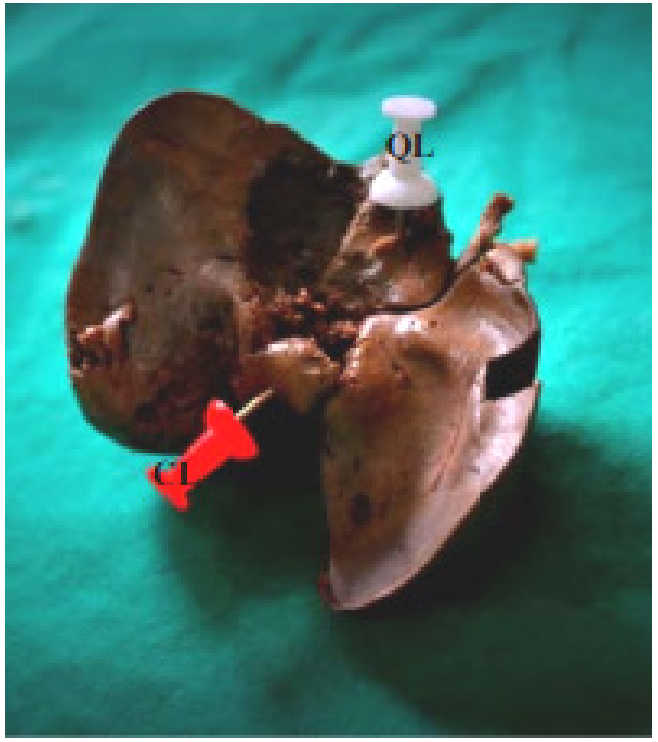

Fig. 1: Photograph of dissected fetal liver showing Quadrate lobe (QL) and Caudate lobe (CL)

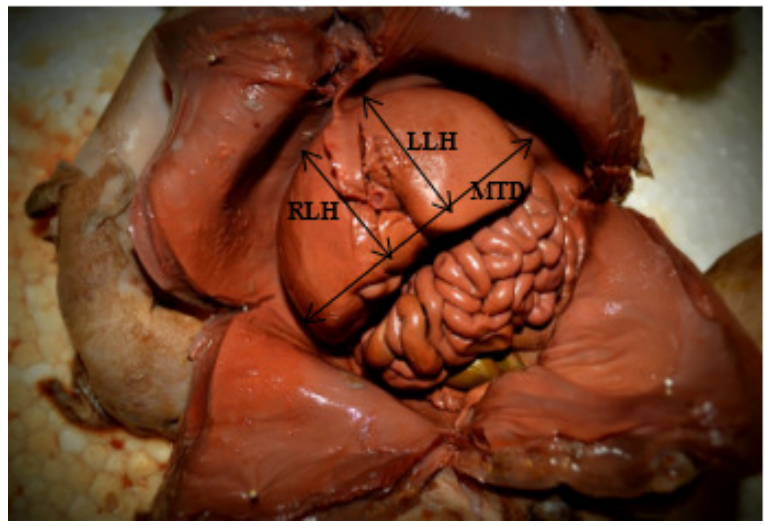

Fig. 2: Photograph of dissected fetal liver showing liver Right lobe height (RLH), Left Lobe height (LLH) and maximum transverse diameter (MTD)

\section{OBSERVATIONS AND RESULTS}

The various gross measurements of fetal liver were studied. The measurement showed marked variations of its length and breadth; however a tendency of increase in various parameters was established with increasing CRL.

The maximum liver transverse diameter (MLTD) was plotted against CRL and showed that the MLTD corresponded well to the increasing CRL (Fig. 3). The transverse diameter of right and left lobes and the height of the right lobe of liver increased in relation to $\mathrm{CRL}$ of fetus. When the transverse diameter of right lobe compared with that of left lobe in relation to CRL, it has been noticed that there was increasing tendency of transverse diameter of right lobe. The left lobe transverse diameter was almost similar from $13.5 \mathrm{~cm}$ up to $28 \mathrm{~cm} \mathrm{CRL}$ and then it became considerably less as compared to the right lobe. This indicates that there is increasing tendency of right lobe enlargement as compared to left lobe (Fig. 3). When height of right lobe and left lobe was compared at $28 \mathrm{~cm} \mathrm{CRL}$, height of right lobe was higher as compared to left lobe. Both right and left lobe height followed almost similar pattern from $6.5 \mathrm{~cm}$ up to $25 \mathrm{~cm} \mathrm{CRL}$, after that at 28 $\mathrm{cm} \mathrm{CRL}$, a difference of height of right and left lobe was observed.

When observation of length and breadth where graphed for quadrate, its breadth did show a variation till $18.5 \mathrm{~cm}$ CRL however, after $19 \mathrm{~cm}$ CRL both its length and breadth followed almost similar pattern running parallel in relation to CRL (Fig. 4). When its length and breadth were graphed, we did not notice much of the difference in early part of observation, however; its breadth was found to be increased subsequently. When length and breadth of caudate lobe were graphed together initially its length shows a tendency of increasing pattern, but after $17 \mathrm{CRL}$ both length and breadth grew almost parallel to the base line. When measurement of length of quadrate and caudate lobes were compared together, marked variation was observed (up and down) in the caudate lobe length from $6.5 \mathrm{CRL}$ to $16 \mathrm{~cm} \mathrm{CRL}$, whereas a gradual rise in tendency was observed in the length of quadrate lobe as compared to the length of the caudate lobe. At $28 \mathrm{~cm} \mathrm{CRL}$ the quadrate lobe was slightly more as compared to caudate lobe length (Fig. 4). 


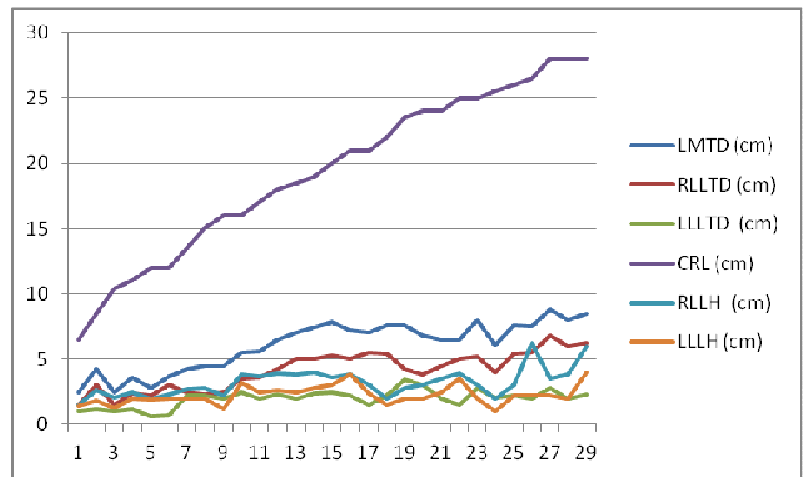

Fig. 3: Graph showing liver maximum transverse diameter (LMTD), right lobe liver transverse diameter (RLLTD), crown rump length (CRL), right lobe liver height (RLLH), left lobe liver transverse diameter (LLLTD), left lobe liver height (LLLH)

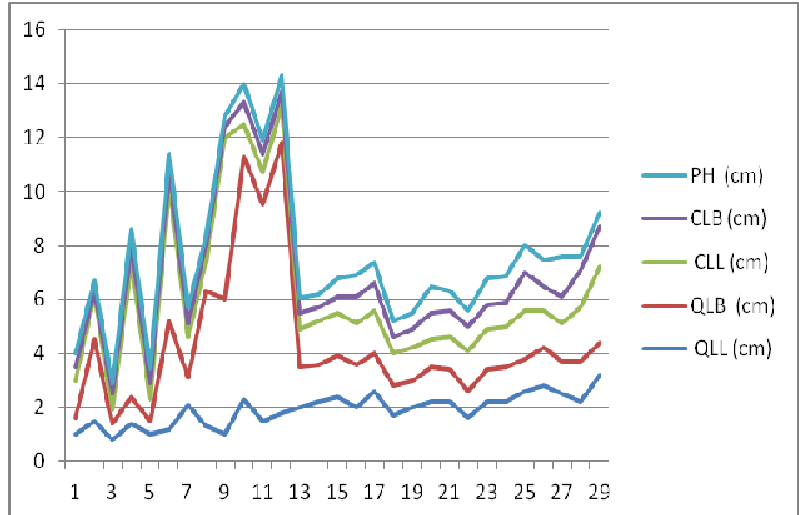

Fig. 4: Graph showing Caudate liver lobe length (CLL), Caudate liver lobe breadth (CLB), Quadrate liver lobe length (QLL), Quadrate liver lobe breadth (QLB), length of porta hepatis (PH)

\section{DISCUSSION}

Adult liver is an abdominal organ mainly localized to right hypochondrium with minimal extension on to left side however fetal liver is markedly different in different trimesters of fetal life. The liver shows different shape in different trimester varying from triangular, square, trapezoid to rectangular type, while near term fetus resembles adult shape [5]. Lee et al. (2003) measured the thickness, height and width of 12 late stage human fetus and found that the fetal liver shows great individual variation with fetal liver showing ellipsoid or oval surface while adult liver was triangular [6]. The marked variations in different parameters may occur because of expansion of abdominal capacities and organization of various viscera which require to be accommodated in the abdominal cavity. The present study has been done keeping in view of the development of liver during fetal period. Gross studies on fetal liver were found to be few in literature.

Various anatomical variations, congenital anomalies and pathologies are associated with intrauterine life. Knowledge of normal rate of growth of various liver parameters is essential to find out these causes and therefore has marked clinical significance. In previous studies, fetal liver length were studied using ultrasound on fetuses older than 2 nd trimester $[7,8]$. In our study, however, we measured, height, transverse diameter of left, right, caudate and quadrate lobe between CRL corresponding to 2.5 and 5.6 weeks of gestation. All these parameters show largely a linear increase with gestational age.

In our study, height of both right and left lobe of liver showed almost similar pattern of increase from $6.5 \mathrm{~cm}$ upto $25 \mathrm{~cm} \mathrm{CRL}$, while at $28 \mathrm{~cm}$ right lobe of liver was found to have greater height. Fetal liver height shows marked difference in various pathologies, in fetuses with gestational diabetic mothers it was found to be higher than normal [4], while it was markedly lower in small-for gestational age (SGA) foetuses [9],emphasizing importance of knowing the normal range of the fetal liver size [5].

The maximum liver transverse diameter (MLTD) in our study corresponded well to the increasing CRL. Both right and left lobe transverse diameter increased in relation to CRL but in later stage, right lobe transverse diameter exceeded that of left side. Fetal liver length has marked clinical significance. Tongprasert et al. (2011) studied on 640 normal pregnant women between 14 and 40 weeks of gestation and found that fetal liver length was gradually increased with gestational age and fetal liver length may be a useful tool in assessment for some fetal pathologic conditions like fetal anemia [10].

Albay et al. (2005) detected a linear increase in the width of the left and right lobes with respect to gestational age and found that ratio between right and left lobe sizes did not show significant change throughout the gestation [5].

Our study showed increasing tendency of right lobe enlargement as compared to left lobe especially in later stage of fetal growth. Baruah \& Choudhury (2013) stated that the left lobe of liver is large in fetal liver [1]. The height of both lobes increased during growth however right lobe showed more growth as compared to left lobe. 
Quadrate lobe showed variation in breadth early in our study, as compared to length which almost showed almost similar pattern running parallel in relation to CRL. The length and breadth of caudate lobe showed a tendency of increasing pattern early up to $17 \mathrm{~cm}$ CRL. Not much data was found on the anthropometric measurement of caudate and quadrate lobe measurement. Albay et al. (2005) demonstrated a high positive correlation between the size of the caudate and quadrate lobes and gestational age [5].

Fetal liver get markedly affected in various pathology like intrauterine growth retardation, gestational diabetes, infections and other conditions and remains an important parameter in there follow-up as shown by different studies [4,11]. This study highlighted that various liver parameters increase in relation to CRL but individual parameters show marked variation and their proper understanding is required to rule out any anatomical variation, congenital anomaly or pathology.

\section{Conflict of interest}

All authors have none to declare.

\section{REFERENCES}

1. Baruah P, Choudhury PR. Tongue-like elongation of the left lobe of liver. OA Case Reports. 2013; 2(17):161.

2. Murao F, Takamiya O, Yamamoto K, Iwanari O. Detection of intrauterine growth retardation based on measurements of size of the liver. Gynecol Obstet Invest. 1990; 29: 26-31.
3. Roberts $A B$, Mitchell JM, Pattison NS. Fetal liver length in normal and isoimmunized pregnancies. Am J Obstet Gynecol. 1989; $161: 42-6$.

4. Fleischer AC, Manning FA, Jeanty $P$, Romero R. Sonography Principles of Obstetrics and Gynecology and Clinical Applications. In: Yuksel A, Turkish editor. Ankara, Turkey: 5th press; 2000. p. 109-130, 411-32.

5. Albay S, Malas MA, Cetin E, Cankara N, Karahan N. Development of the liver during the fetal period.Saudi Med J. 2005; 26(11):1710-5.

6. Lee SD, Kim CY, Cho YH, Fujiwara D, Murakami G, Mutsumura H, Lee SK, Kim JH, Jin ZW, Cho BH. Morphometrical data of size and shape of late stage human fetal liver, including these of intrahepatic vessel. Some prenatal and postnatal developmental consideration. Korean J HBP Surg. 2003; 7:12-18.

7. Murao F, Takamori H, Aoki S, Hata K, Hata T, Yamamoto K. Ultrasonographic measurement of the human fetal liver in utero. Gynecol Obstet Invest. 1987; 24: 145-150.

8. Murao F, Takamori H, Hata K, Hata T, Kitao M. Fetal liver measurements by ultrasonography. Int J Gynaecol Obstet. 1987; 25: 381-385.

9. Roberts $A B$, Mitchell JM, McCowan LM, Barker $S$. Ultrasonographic measurement of liver length in the small forgestational-age fetus. Am J Obstet Gynecol. 1999; 180:634-8.

10. Tongprasert F, Srisupundit K, Luewan S, Tongsong T. Normal length of the fetal liver from 14 to 40 weeks of gestational age. J. Clin. Ultrasound. 2011; 39: 74-7.

11. Ghidini A, Sirtori M, Romero R, Yarkoni S, Solomon L, Hobbins JC. Hepatosplenomegaly as the only prenatal finding in a fetus with pyruvate kinase deficiency anemia. Am J Perinatol. 1991; 8: 44-46. 